\title{
Biotechnological Assessment of Culture Conditions on the Stress-Induced Carotenoid Production of Dunaliella salina and Growth Kinetics of Chlorophyceae Microalgae Strains
}

\author{
Cíntia Jesus Almeida ${ }^{1,2}$ \\ https://orcid.org/0000-0002-9101-6184
}

Diego de Carvalho Carneiro'

https://orcid.org/0000-0002-2258-8197

\section{Emerson Andrade Sales ${ }^{2}$}

https://orcid.org/0000-0002-9607-7285

\section{Suzana Telles da Cunha Lima ${ }^{1 *}$ \\ https://orcid.org/0000-0002-9099-324X}

${ }^{1}$ Federal University of Bahia, Institute of Biology, Salvador, Bahia, Brazil; ${ }^{2}$ Federal University of Bahia, Escola Politécnica, Salvador, Bahia, Brazil.

Received: 2019.04.01; Accepted: 2020.02.13

*Correspondence: stcunhalima@ufba.br

\section{HIGHLIGHTS}

- The aeration of atmospheric air condition presented the best performance and a growth rate increase of $35-56 \%$.

- The intense light condition rendered a 53\% increase in carotenoid production.

- The nitrogen deprivation condition yielded a lower cellular population but a carotenoid production similar to the control.

Abstract: Microalgae are potential sources of a wide range of bioproducts. It is essential to choose the proper microalgae strain and culture condition to achieve an efficient production. The production yield of carotenoids by Dunaliella salina under the stress-induced culture conditions of nitrogen deprivation and excessive light intensity was evaluated. Also, a survey at laboratorial scale of the growth kinetics under different culture conditions of photoperiod, aeration, and agitation was performed for the seven species of green microalgae Ankistrodesmus fusiformis, Chlamydocapsa bacillus, Desmodesmus brasiliensis, Kirchneriella lunaris, Pseudokirchneriella subcapitata, and Scenedesmus obliquus. As a result, aeration of atmospheric air is enough to improve the growth kinetics of the seven species studied. Production of carotenoids was enhanced under stress by excessive light intensity. Although $D$. salina does not grow effectively under nitrogen deprivation, this stress condition may be used to quickly stimulate carotenoid production once the culture reaches a high cellular population.

Keywords: growth rate; biomass productivity; nitrogen deprivation; light intensity stress; carotene production. 


\section{INTRODUCTION}

The proper devise of photoperiods in microalgae cultures can have a significant impact on their economical cost-benefit by means of energy demand and lipid productivity improvement. The photosynthetic metabolism of microalgae requires primarily $\mathrm{CO}_{2}$ and light sources in which the sunlight presents the best cost-benefit but depends on the weather, climate, and day/night cycles. On the other hand, the light and dark reactions of photosynthesis permit the adjustment of the microalgal productivity under artificial illumination according to the photoperiod [1,2].

Agitation is used in microalgae cultures to homogenize and ensure to most cells access to $\mathrm{CO}_{2}$, nutrients and light, as well as to facilitate the removal of $\mathrm{O}_{2}$. An excessive sheer stress from mechanical agitation can reduce growth and productivity and cause cellular damage. The resistance of microalgae species to sheer stress depends on their cell wall composition and structure [3]. Aeration supplies the culture with $\mathrm{CO}_{2}$, controls its $\mathrm{pH}$, and also facilitates removal of $\mathrm{O}_{2}$ and homogenization. $\mathrm{CO}_{2}$ fixation is directly related to biomass productivity and depends on the flow, distribution and concentration of this gas in the culture system [4,5].

Among the products of high aggregated value and biotechnological interest are the carotenoid pigments. A species that stands out for its great potential for the synthesis of carotenoids is Dunaliella salina. Stressinduced changes in the cellular physiological balance generate free radicals that encourages synthesis of $\beta$ carotene to protect the cells. When stimulated by adverse conditions of growth such as intense light and limiting nitrogen, $D$. salina changes its metabolism to accumulate considerable amounts of $\beta$-carotene, displaying a progressive yellow color, which may comprise up to $14 \%$ of its dry weight $[6,7]$.

Carotenogenesis takes place in the chloroplasts, where the primary carotenoids function in light harvesting and protection of the photosynthetic system from excess light. The secondary carotenoids do not play a role in photosynthesis, and they are usually accumulated in cytoplasmic membrane vesicles as a response to stress. The first step of carotenoid synthesis involves the enzyme phytoene synthase that condensates geranylgeranyl pyrophosphate molecules to form phytoene. Next, desaturase enzymes perform successive desaturation reactions to form lycopene. Here, intense light and limiting nitrogen conditions can increase the number of desaturase enzymes, consequently increasing carotenoid production. Finally, lycopene cyclases catalyze the cyclization of both terminal carbons of lycopene to form $\alpha$-carotene and $\beta$ carotene, which may be subjected to derivative reactions to form other carotenoids [8].

In this study, the influence of aeration, photoperiod and agitation culture conditions on the growth of a set of green microalgae strains is presented. The influence of the stress-inducing conditions of intense light and nitrogen deprivation on carotenoid production by $D$. salina is also described. Perspectives on how those conditions can be used to improve growth and carotenoid production are suggested. This study may be resourceful for choice-making of species and culture conditions in other researches to improve microalgae production.

\section{MATERIALS AND METHODS}

\section{Growth kinetics and biomass productivity}

The green (Chlorophyceae) microalgae species Ankistrodesmus fusiformis, Chlamydocapsa bacillus, Desmodesmus brasiliensis, Kirchneriella lunaris, Pseudokirchneriella subcapitata, and Scenedesmus obliquus were obtained from a collection maintained by the LaBBiotec (Laboratory of Bioprospection and Biotechnology), at the Institute of Biology, Federal University of Bahia, Brazil. This collection was assembled with microalgae collected and identified from a eutrophic lagoon located at Salvador, Bahia, Brazil. The nutrient-sufficient medium LC Oligo was used to maintain and cultivate the microalgae using fluorescent lamps at room temperature $\left(23 \pm 2{ }^{\circ} \mathrm{C}\right)$ and light intensity of $10 \mu \mathrm{mol} \mathrm{m} \mathrm{m}^{-2} \mathrm{~s}^{-1}$ [9].

Batch mode growth was performed at room temperature using Erlenmeyer flasks with $80 \mathrm{~mL}$ of medium and $5 \%$ of inoculum in the exponential phase. The growth kinetics was monitored every 2 days during 10 days of culture by measuring the optical density at $680 \mathrm{~nm}$ (OD680) in duplicate or triplicate using a spectrophotometer (UNICAM ${ }^{\circledR}$ He $\lambda$ ios $\varepsilon$ ). Constant (24L:OD) and intermittent (12L:12D) photoperiods of light (L) and dark (D) cycles as well as agitation and aeration of atmospheric air at $210-280 \mathrm{~mL} / \mathrm{min}$ were used to compare the growth kinetics by calculating the specific growth rate $(\mu)$ of each microalgae strain using OD680 values with $R^{2}>0.95$ through the following equation:

$$
\mu=\frac{\ln \left(\frac{N_{2}}{N_{1}}\right)}{t_{2}-t_{1}},
$$


Where $N_{1}$ is the first OD680 mean measured at day $t_{1}$, and $N_{2}$ is the last OD680 mean measured at day $t_{2}$ [10].

Biomass productivity was attained by duplicate cultures under room temperature, photoperiod 12L:12D, and aeration of atmospheric air at $230-370 \mathrm{~mL} / \mathrm{min}$ in Erlenmeyer flasks with $250 \mathrm{~mL}$ of medium and $5 \%$ of inoculum in the exponential phase. Every 2 days during 16 days of culture, aliquots of $15 \mathrm{~mL}$ were collected and filtered under vacuum pump using $0.7 \mu \mathrm{m}$ filters. The resulting filters with wet biomass were gathered and maintained at $-20^{\circ} \mathrm{C}$ until their drying in an incubator at $80^{\circ} \mathrm{C}$ with airflow renewal during $14 \mathrm{~h}($ Tecnal $\Theta$ ). The biomass dry weight per volume of aliquot $(\mathrm{g} / \mathrm{L}$ ) were plotted against time (days), and those with resulting $\mathrm{R} 2>0.95$ were later used to calculate the biomass productivity $(\mathrm{Px})$ in the exponential phase by the following equation:

$$
P x=\frac{X_{2}-X_{1}}{t_{2}-t_{1}}
$$

where $X_{1}$ is the first biomass dry weight measured at day $t_{1}$, and $X_{2}$ is the last biomass dry weight measured at day $t_{2}$ [10]. The specific growth rate of the cultures used to measure the biomass productivity was calculated by the biomass dry weight values instead of the OD680 measurements for N1 and N2 in Equation 1.

\section{Stress-inducing culture conditions}

For this study, a $D$. salina stock culture was obtained from the LABEC (Laboratory of Bioenergy and Catalysis) located at the Polytechnic School, Federal University of Bahia, Brazil. Cultivation was performed in $2 \mathrm{~L}$ of Conway medium [11] at regular conditions using fluorescent lamps at light intensity of $70 \mu \mathrm{mol} \mathrm{m}^{-2}$ $\mathrm{s}^{-1}$ and room temperature. Agitation was achieved by constantly bubbling sterile air using a $0.20 \mu \mathrm{m}$ filter, ensuring the turbulence and aeration of the media inside the flask. Equal volumes of this cultivation were used to inoculate the stress-inducing culture conditions.

Triplicate cultures for the control and the stress-inducing conditions of intense light and nitrogen deprivation were performed. The control was prepared in regular conditions, with no limiting concentrations of nutrients. The nitrogen deprived culture condition was prepared using Conway medium without addition of $\mathrm{NaNO}_{3}$. In order to nitrogen deprivation act as only stressing factor, the Erlenmeyer flasks were transferred to the shelves with the same luminosity, temperature and aeration of those applied to the control. For the light intensity stress condition, no limiting nutrient was used and the Erlenmeyer flasks were kept on shelves illuminated by both sides with fluorescent lamps at room temperature.

Growth monitoring was carried out by microscopy cell counting in Neubauer chamber, fixing the mobile cells of $D$. salina with Transeau, formulated with water, alcohol, and formaldehyde at the proportion 6:3:1, respectively [12]. Measurements of the $\mathrm{pH}$ of the cultures were performed during growth monitoring, and the specific growth rate was calculated by Equation 1 using $t_{1}$ and $t_{2}$ at 0 and 6 days, respectively.

\section{Extraction of carotenoids}

Carotenoids were extracted from $D$. salina as adapted from the method described by Sedmak and coauthors [13]. Briefly, the procedure is based on the maceration of $2 \mathrm{~mL}$ of culture samples, followed by centrifugation at 4,000 rpm for 5 minutes in falcon tubes. After disposing the supernatant, $1 \mathrm{~mL}$ of dimethyl sulfoxide (DMSO) was added, the pellet was vortexed for 20-30 seconds and left for 5 minutes to react. For separation of carotenoids, $0.1 \mathrm{~mL}$ of $0.01 \mathrm{M}$ sodium phosphate buffer at $\mathrm{pH} 7.0$ and $1 \mathrm{~mL}$ of acetone were added to the mixture. The tubes were vortexed again for 30-40 seconds to mix the phases, which were then separated by centrifugation at $4,000 \mathrm{rpm}$ for 5 minutes. The procedure was repeated until total extinction of color. The supernatants were collected and covered with aluminum foil to prevent exposure to light. The synthetic Trans $\beta$-carotene Type I (Sigma-Aldrich ${ }^{\circledR}$ ) with $93 \%$ purity was used as standard at different concentrations for quantification by linear regression of the extracted carotenoids from $D$. salina through absorbance readings of sample and standards at $450 \mathrm{~nm}$.

\section{Thin-layer chromatography}

Thin-layer chromatography was performed to confirm the composition of pigments from the carotenoid extracts. The stationary phase was prepared in glass plates with a thin layer of silica gel previously dried at $70{ }^{\circ} \mathrm{C}$. The mobile phase or eluent solution was composed by chloroform, acetone and acetic acid at the proportion 95:5:1, respectively. A small volume of synthetic $\beta$-carotene (standard) and samples of pigments extracted from the microalga were applied to the silica plates and incubated in a beaker with the eluent 
solution, where a solvent shift was observed. After drying for about 5 minutes at room temperature, the plate was placed in a container with solid iodine until complete revelation.

\section{RESULTS AND DISCUSSION}

\section{Influence of culture conditions on the growth kinetics of microalgae}

The growth rate of microalgae presents significant differences between the initial (0-3 days) and later (310 days) periods of the exponential phase, as demonstrated by Krzeminska and coauthors [14]. In addition, the light intensity used in this study does not significantly influences on growth, as described by Ota and coauthors [15]. As shown in Figure 1, all species of microalgae presented an increase in the growth rate between 35 and $56 \%$ under aeration when compared to the values from the same photoperiod (12L:12D) without aeration during the initial period of the exponential phase (10 days of culture). An improvement of $53 \%$ in the growth was observed for $A$. fusiformis under agitation and $30 \%$ for $D$. brasiliensis under constant photoperiod. In addition, agitation seems to decrease the growth rate of $C$. bacillus in $58 \%$. This result can be explained by the ability of $C$. bacillus to form biofilm since agitation can cause its disruption.

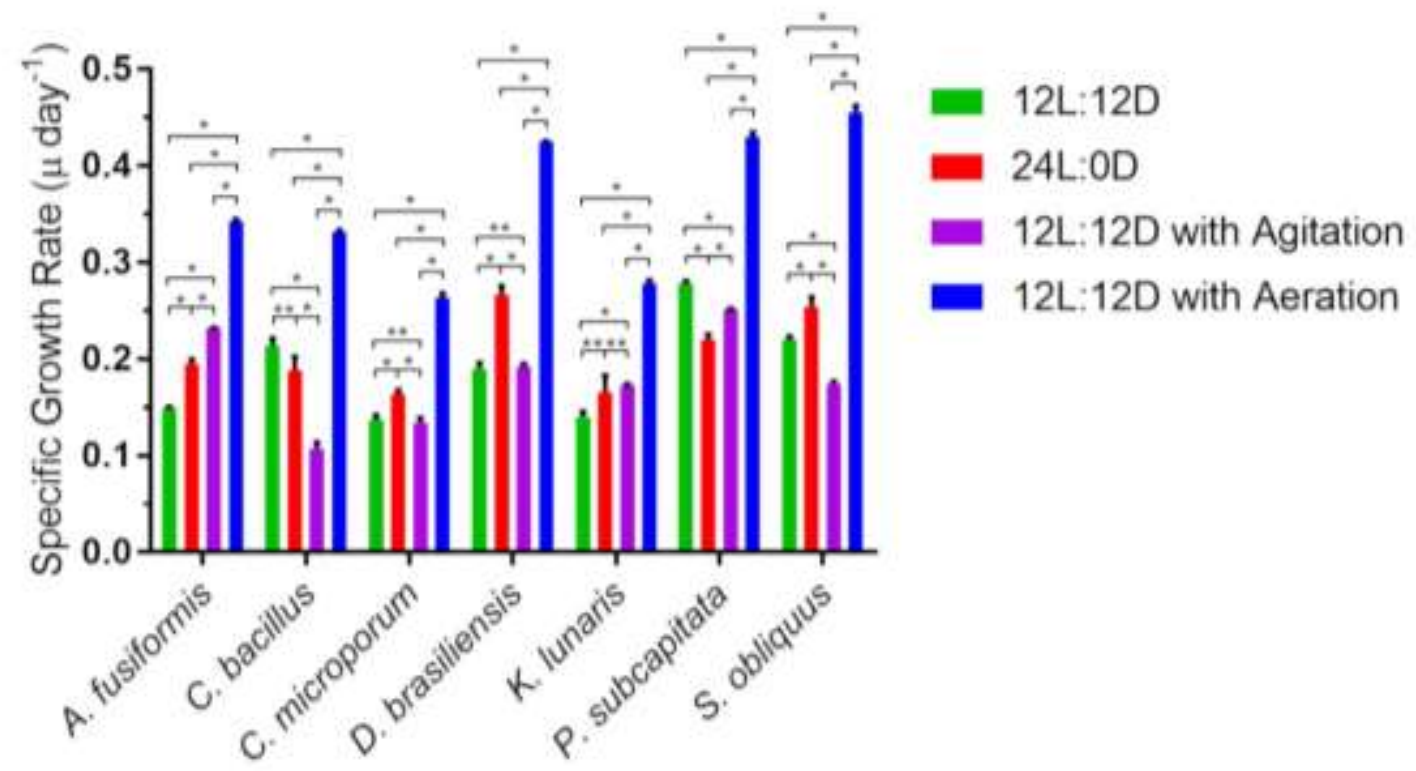

Figure 1. Specific growth rate of each microalgae strain under different conditions of batch mode cultures. $\left({ }^{*}\right) \mathrm{T}$-test with $\left.p<0.05 ;{ }^{* *}\right)$ T-test with $p>0.05$.

The biomass productivities and the specific growth rates calculated from the values of biomass dry weight for longer periods (more than 10 days) are shown in Table 1 with their respective standard deviations (SDs). The highest Px was observed for $D$. brasiliensis, while $K$. lunaris, $C$. bacillus and $C$. microporum presented Px values of about $56 \%$ lower. The other species were found with biomass productivities between 21 and $36 \%$ lower than $D$. brasiliensis. Similar results were found in other studies for $S$. obliquus and $K$. lunaris $[14,16]$.

Table 1. Biomass productivity and specific growth rate from the biomass dry weights of the microalgae strains

\begin{tabular}{lll}
\hline Microalgae Strain & $\operatorname{Px}\left(\mathrm{g} \mathrm{L}^{-1} \mathrm{day}^{-1}\right)$ & $\mu\left(\mathrm{day}^{-1}\right)$ \\
\hline Ankistrodesmus fusiformis & $0.09 \pm 0.00$ & $0.19 \pm 0.03$ \\
Chlamydocapsa bacillus & $0.06 \pm 0.01$ & $0.15 \pm 0.01$ \\
Coelastrum microporum & $0.06 \pm 0.00$ & $0.13 \pm 0.04$ \\
Desmodesmus brasiliensis & $0.14 \pm 0.04$ & $0.21 \pm 0.02$ \\
Kirchneriella lunaris & $0.06 \pm 0.01$ & $0.17 \pm 0.03$ \\
Pseudokirchneriella subcapitata & $0.11 \pm 0.02$ & $0.21 \pm 0.01$ \\
Scenedesmus obliquus & $0.09 \pm 0.01$ & $0.20 \pm 0.05$ \\
\hline
\end{tabular}


According to the records of Nascimento and coauthors. [17], close values of biomass productivity and specific growth rate (shown in Table 1) were also registered for $C$. microporum, $D$. brasiliensis, $K$. lunaris, $P$. subcapitata, and S. obliquus from their culture system with aeration of atmospheric air enriched with $2 \%$ of $\mathrm{CO}_{2}$. Therefore, $\mathrm{CO}_{2}$ fixation was not significantly affected by the enhancement in $\mathrm{CO}_{2}$ concentration. $A$. fusiformis presented a $\mu$ value close to the one shown in Figure 1 but a Px value $62 \%$ lower. On the other hand, $C$. bacillus presented a $\mu$ value $56 \%$ lower and a Px value $81 \%$ lower, which may be explained by the distinct methods of culture and growth monitoring used in each study. For this species, the injection of $2 \%$ $\mathrm{CO}_{2}$ may also have increased its growth and biomass productivity.

Maroneze and coauthors [1] demonstrated that the best photoperiod for $S$. obliquus is 22L:2D in which the $2 \mathrm{~h}$ of dark divided in $24 \mathrm{x}$ during the day rendered a better cost-benefit lipid production, resulting in a reduction of $40.7 \%$ in energy demand. The short photoperiod of $0.5 \mathrm{~L}: 0.5 \mathrm{D}$ (in seconds) presented the best cost-benefit for biomass productivity, rendering 33\% reduction in energy demand. Anjos and coauthors. [4] showed that the optimization of microalgal cultures can be an efficient strategy to increase $\mathrm{CO}_{2}$ fixation. This study demonstrated a maximum biomass productivity of $1.3 \mathrm{~g} \mathrm{~L}^{-1}$ day ${ }^{-1}$ by Chlorella vulgaris in a culture system with aeration of atmospheric air enriched with $6 \% \mathrm{CO}_{2}$ at $0.4 \mathrm{vvm}$.

\section{Stress-inducing production of carotenoids by D. salina}

$D$. salina is able to achieve a maximum growth rate at $127 \mu \mathrm{mol} \mathrm{m} \mathrm{m}^{-2} \mathrm{~s}^{-1}$ as indicated by Giordano and Beardall. [18]. Although the effects of salinity, light and temperature on growth is complex, light is the less important parameter. On the other hand, García-González and coauthors [19] was able to grow D. salina in a semi-continuous mode at $200 \mu \mathrm{mol} \mathrm{m}^{-2} \mathrm{~s}^{-1}$, reaching a specific growth rate of about $0.18 /$ day.

Figure 2 shows the culture settings of $D$. salina under control and stress-inducing conditions (a-c) as well as the appearance of the microalgal cells (d-f). The growth curves of each condition are shown in Figure 3. The specific growth rate in the exponential phase from the control, intense light and nitrogen deprivation conditions are $0.25,0.33$ and 0.14 , respectively.
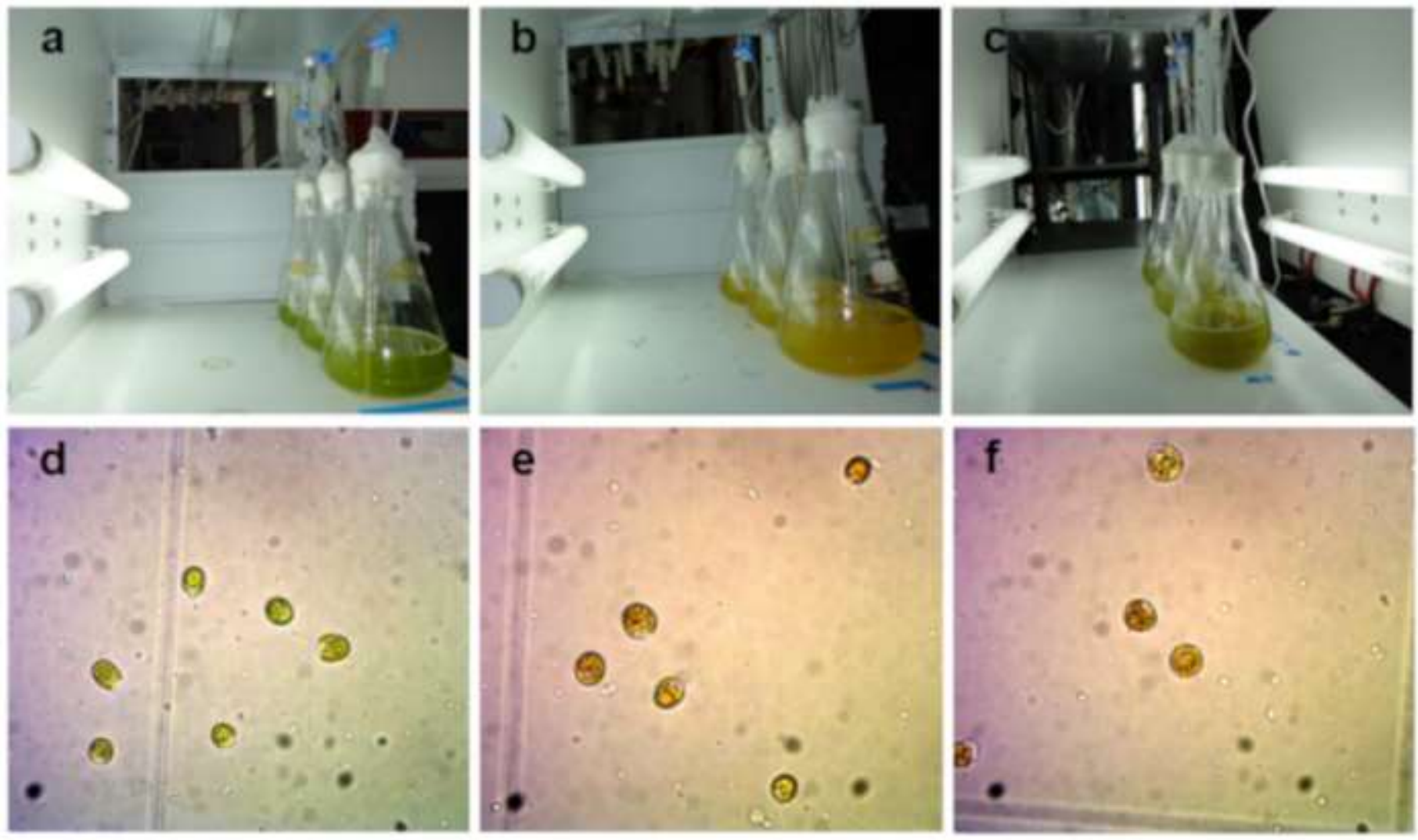

Figure 2. Culture settings and cellular appearance of $D$. salina under the culture conditions. (a, d) Control; (b, e) Nitrogen deprivation; (c, f) Intense light. 


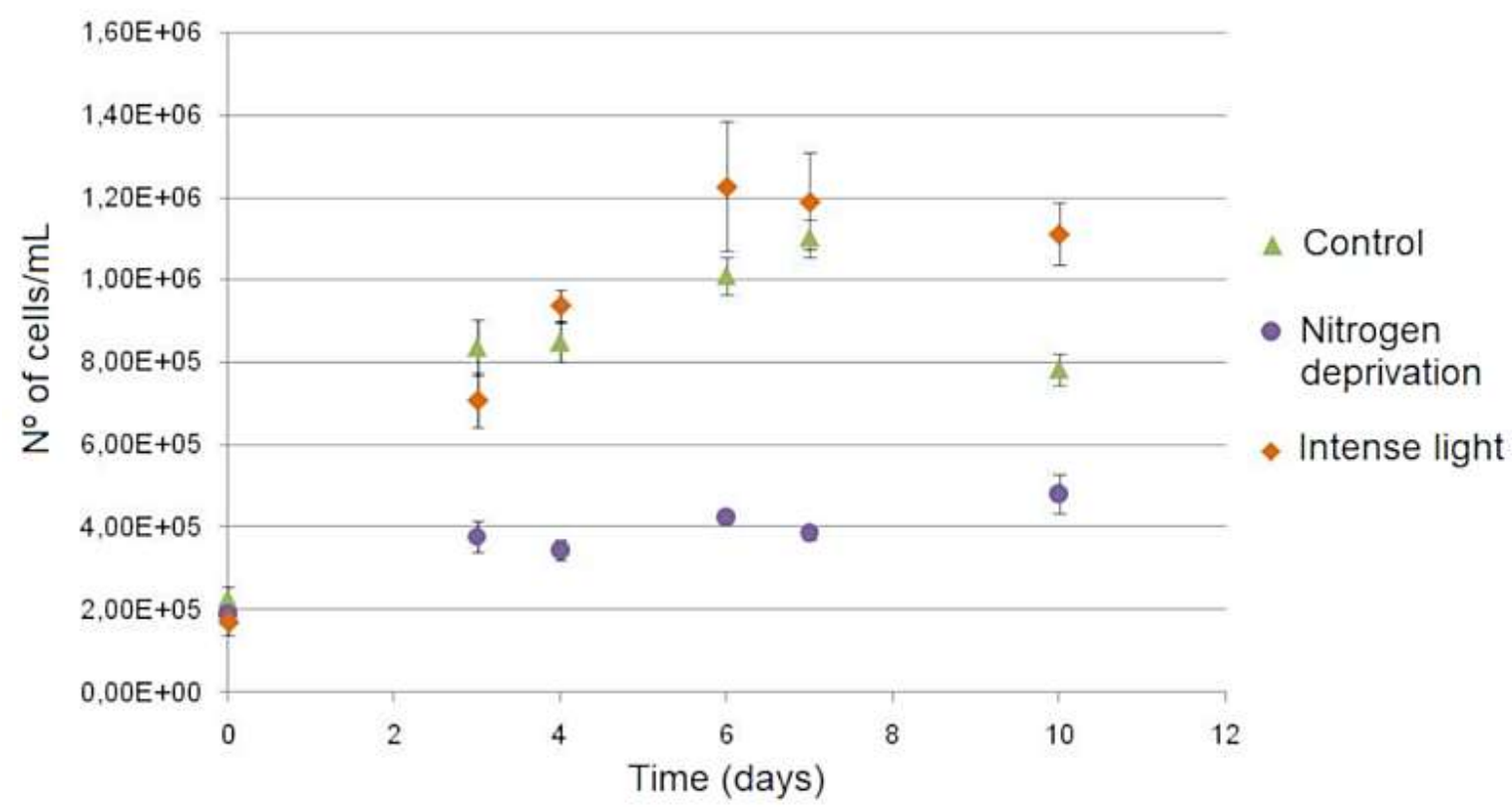

Figure 3. Growth curves of $D$. salina under control and stress-inducing conditions.

The inability of the nitrogen deprivation condition to provide a satisfactory nutritional medium significantly decreased the growth rate of $D$. salina. However, a faster change of color due to carotenoid production was observed for the nitrogen deprivation condition. On the sixth day of cultivation, the predominance of yellow color was already observed despite the small concentration of cells present in the culture.

The $\mathrm{pH}$ values for the three conditions did not exceeded 9.2 and framed within the optimum $\mathrm{pH}$ limits for D. salina (7.5-9.0), according to Khalil and coauthors [20]. Increase in pH to values above 8.0 is due to the natural consumption of $\mathrm{CO}_{2}$ from the medium [21]. The highest $\mathrm{pH}$ was observed in the culture under intense light stress, which may be explained by an increased consumption of dissolved $\mathrm{CO}_{2}$ due to its higher cellular population.

As shown in Figure 4, the profile and retention factors of the extracted pigments from the control and stress-inducing conditions were similar to the standard, confirming the extraction and purity similarity to standard of the carotenoids from $D$. salina by the methodology used in this study. The amount of carotenoids extracted from each condition can be seen in Figure 5. As indicated by t-test analyses, the chances for the carotenoid concentrations between control and intense light conditions as well as between nitrogen deprivation and intense light conditions to be equal are not significant $(p<0.05)$. However, they were significantly equal $(p>0.05)$ between the control and nitrogen deprivation conditions.
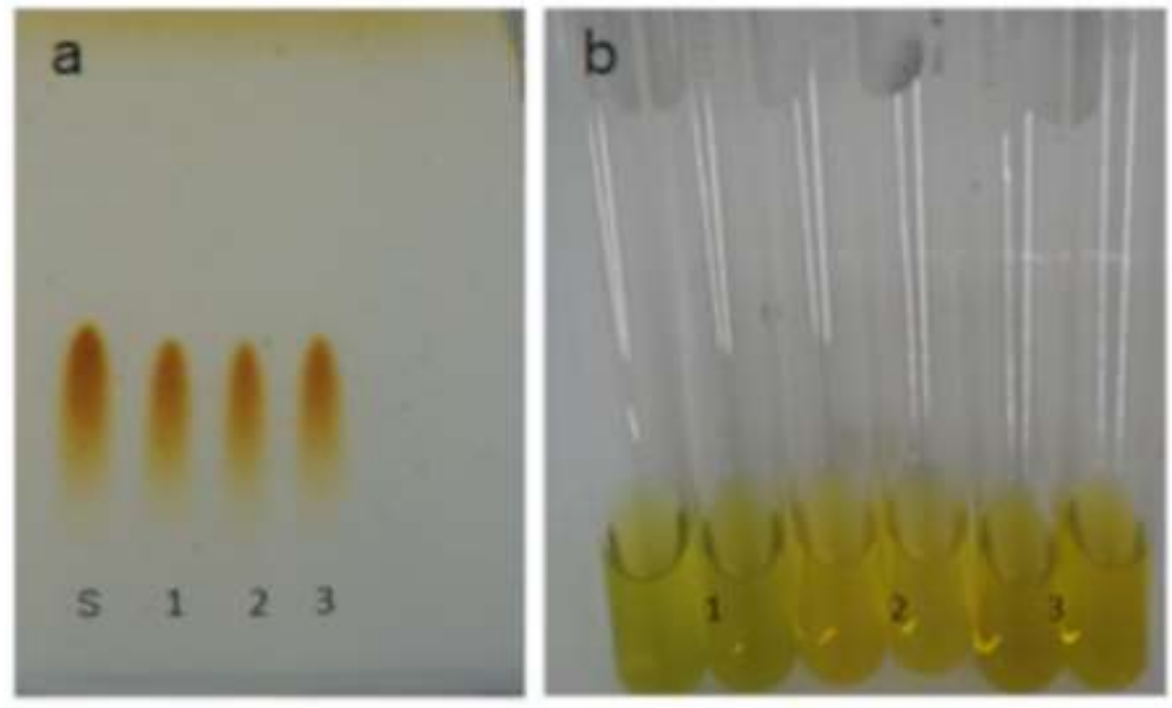

Figure 4. Profile of the extracted carotenoid pigments from D. salina. (a) Chromatogram; (b) Samples; (S) Standard; (1) Control; (2) Nitrogen deprivation; (3) Intense light. 
As can be seen in Figures 3 and 5, since the growth curves and specific growth rates of the control and intense light conditions were similar, the enhancement of carotenoid concentration under intense light can be associated to this stress-inducing condition, which rendered in this study an increase of $53 \%$ in the production of this pigment by $D$. salina. In addition, because the growth rate and cellular population of the nitrogen deprivation condition was lower than the control but presented significant similar concentration of carotenoids, it can also be associated to this condition an improvement in carotenoid production.

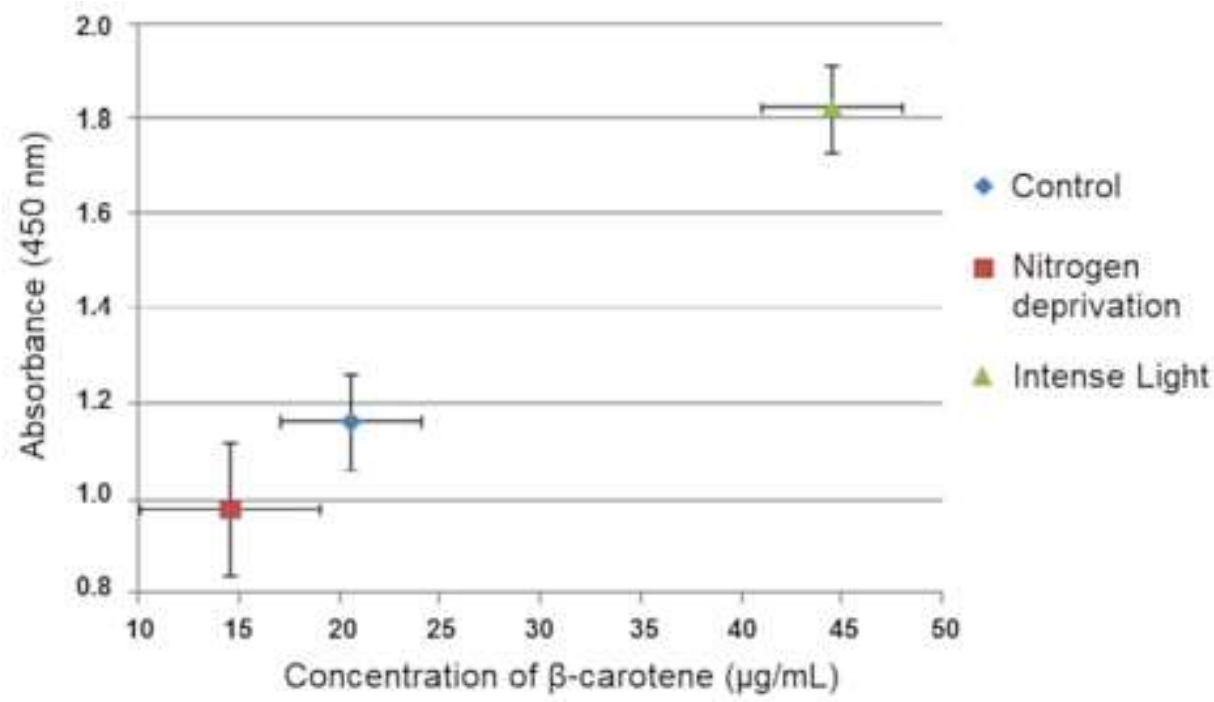

Figure 5. Concentrations of carotenoids extracted from $D$. salina under control and stress-inducing conditions.

\section{CONCLUSION}

Aeration of atmospheric air without $\mathrm{CO}_{2}$ enrichment was able to significantly increase the growth kinetics of the green microalgae species, so it may be enough to satisfactorily enhance the cost-benefit of production systems involving the seven strains studied. In addition, inducing stress by using intense light exposure can improve production of carotenoids by $D$. salina. On the other hand, nitrogen deprivation is not able to sustain cellular division in order to increase carotenoid production along a biomass growth, but it can enhance the production speed. Once a $D$. salina culture reaches a high cellular density under optimal conditions, it can be suggested the use of nitrogen deprivation for a faster and higher production of carotenoids. Overcoming the challenges of choosing the proper species, and designing culture systems by improving growth kinetics and product yield are essential steps to improve microalgae production.

Funding: This research received no external funding.

Acknowledgments: The authors would like to acknowledge CAPES (Coordenação de Aperfeiçoamento de Pessoal de Nível Superior) and FAPESB (Fundação de Amparo à Pesquisa do Estado da Bahia) for the support.

Conflicts of Interest: The authors declare no conflict of interest.

\section{REFERENCES}

1. Maroneze MM, Siqueira SF, Vendruscolo RG, Wagner R, De Menezes CR, Zepka LQ, Jacob-lopes E. The role of photoperiods on photobioreactors - A potential strategy to reduce costs. Bioresour Technol. 2016;219:493-499.

2. Srirangan S, Sauer ML, Howard B, Dvora M, Dums J, Backman P, Sederoff H. Interaction of Temperature and Photoperiod Increases Growth and Oil Content in the Marine Microalgae Dunaliella viridis. PLOS ONE. 2014; 10: e0127562.

3. Wang C, Lan CQ. Effects of shear stress on microalgae - A review. Biotechnol Adv. 2018; 36: 986-1002.

4. Anjos M, Fernandes BD, Vicente AA, Teixeira JA, Dragone G. Optimization of $\mathrm{CO}_{2}$ bio-mitigation by Chlorella vulgaris. Bioresour Technol. 2013; 139: 149-154.

5. Huang Y, Zhao S, Ding Y, Liao Q, Huang Y, Zhu X. Optimizing the gas distributor based on $\mathrm{CO}_{2}$ bubble dynamic behaviors to improve microalgal biomass production in an air-lift photo-bioreactor. Bioresour Techol. 2017; 233: 84-91.

6. $\quad$ Pisal DS, Lele SS. Carotenoid production from microalga, Dunaliella salina. Indian J Biotechnol. 2005; 4: 476483. 
7. Boussiba S, Fan L, Vonshak A. Enhancement and determination of astaxanthin accumulation in green alga Haematococcus pluvialis. In: Lester P. Methods in Enzymology. New York: Academic Press; 1992. p. 386-391.

8. Henríquez V, Escobar C, Galarza J, Gimpel J. Carotenoids in Microalgae. In: Stange C. Carotenoids in Nature: Biosynthesis, Regulation and Function. Cham: Springer; 2016.

9. Richmond A. Handbook of microalgal culture: biotechnology and applied phycology. Oxford: Blackwell; 2004.

10. Schmidell W, Lima UA, Aquarone E, Borzani W. Biotecnologia Industrial: Engenharia Bioquímia. São Paulo: Bluher; 2008.

11. Walne PR. Experiments in the large scale culture of the larvae of Ostrea edulis. Fishery Investigations. 1966; 25(4): 1-53.

12. Parra OO, Bicudo CEM. Introduccion a la Biologia y Sistematica de las Algas Continentales. Barcelona: Omega; 1995. p. 268.

13. Sedmak JJ, Weerasinghe DK, Jolly SO. Extraction and quantitation of astaxanthin from Phaffia rhodozyma. Biotechnology Techniques. 1990;4:107-112.

14. Krzeminska I, Pawlik-Skowronska B, Trzcinska M, Tys J. Influence of photoperiods on the growth rate and biomass productivity of green microalgae. Bioprocess Biosyst Eng. 2014; 37: 735-741.

15. Ota M, Takenaka M, Sato Y, Lee R, Jr S, Inomata H. Effects of light intensity and temperature on photoautotrophic growth of a green microalga Chlorococcum littorale. Biotechnol Reports. 2015; 7: 24-29.

16. Song M, Pei H, Hu W, Ma G. Evaluation of the potential of 10 microalgal strains for biodiesel production. Bioresour Technol. 2013; 141: 245-251.

17. Nascimento IA, Teles I, Cabanelas D, De Souza CO, Vich DV. Screening Microalgae Strains for Biodiesel Production: Lipid Productivity and Estimation of Fuel Quality Based on Fatty Acids Profiles as Selective Criteria. Bioenerg Res. 2013; 6: 1-13.

18. Giordano M, Beardall J. Impact of environmental conditions on photosynthesis, growth and carbon allocation strategies of hypersaline species of Dunaliella. Global NEST. 2009; 11(1): 79-85.

19. García-González M, Moreno J, Manzano JC, Florencio FJ, Guerrero MG. Production of Dunaliella salina biomass rich in 9-cis - $\beta$-carotene and lutein in a closed tubular photobioreactor. J Biotechnol. 2005; 115: 81-90.

20. Khalil ZI, Asker MM, El-Sayed S, Kobbia IA. Effect of pH on growth and biochemical responses of Dunaliella bardawil and Chlorella ellipsoidea. World J Microbiol Biotechnol. 2010; 26: 1225-1231.

21. Lourenço SO. Cultivo de microalgas marinhas: princípios e aplicações. São Carlos: Rima; 2006. p. 606.

(C) 2020 by the authors. Submitted for possible open access publication under the terms and conditions of the Creative Commons Attribution (CC BY NC) license (https://creativecommons.org/licenses/by-nc/4.0/). 